\title{
Communication
}

\section{Efficient CO-CA transfer in highly deuterated proteins by band-selective homonuclear cross-polarization}

\author{
Veniamin Chevelkov, Karin Giller, Stefan Becker, Adam Lange* \\ Max Planck Institute for Biophysical Chemistry, Göttingen, Germany
}

\section{A R T I C L E I N F O}

Article history:

Received 10 December 2012

Revised 8 February 2013

Available online 15 March 2013

\section{Keywords:}

Cross polarization

Magnetization transfer

Recoupling

Protein resonance assignment

Deuteration

\begin{abstract}
A B S T R A C T
Robust and efficient band-selective magnetization transfer between CO and CA spins can be achieved in highly deuterated solid proteins by dipolar-based homonuclear cross polarization. The approach is designed for moderate magic-angle spinning rates and high external magnetic fields where the isotropic chemical shift difference of $\mathrm{CO}$ and CA considerably exceeds the spinning rate. The most efficient recoupling is achieved when the sum of effective radio-frequency fields on $\mathrm{CO}$ and CA resonances equals two times the spinning rate. This method can be directly implemented in proton-detected versions of interresidual correlation experiments as needed for resonance assignment in protein solid-state NMR spectroscopy.
\end{abstract}

(c) 2013 Elsevier Inc. All rights reserved.

\section{Introduction}

In the last decade solid-state NMR (ssNMR) spectroscopy of rotating solids has progressed into a unique and powerful tool to study structure [1-3] and dynamics [4,5] of biological macromolecules at atomic resolution. To overcome the inherently low sensitivity and resolution, which limits the application of magic-angle spinning (MAS) ssNMR, a number of developments have been made. One promising approach in biomolecular ssNMR is to employ proton detection to make use of the high gyromagnetic ratio of protons [6-9]. These experiments became feasible by employing deuterated samples and/or high sample rotation rates. The approach resulted in a number of successful studies of dynamics [4,5] and structure [9-11] of biomolecules. An essential step in protein NMR studies is the resonance assignment, which requires new or modified experimental approaches in case of proton detected NMR compared to conventionally used carbon detected experiments. There are numerous strategies to assign deuterated proteins in proton-detected experiments using both scalar and dipolar couplings $[9,12-14]$. Recent studies show that proton-detected experiments allow for the assignment of rather large systems, namely the $16 \mathrm{kDa}$ protein SOD [14] and the $21 \mathrm{kDa}$ protein DsbA [12]. One crucial step in many assignment schemes is the magnetization transfer between $\mathrm{CO}$ and CA nuclei, for example in proton-detected experiments of the type $(x) \mathrm{CO}(\mathrm{CA}) \mathrm{NH}$. Pintacuda and coworkers made use of the relatively strong $\mathrm{CO}-\mathrm{CA}$ scalar coupling [14] of $55 \mathrm{~Hz}$ in INEPT-type experiments, while

\footnotetext{
* Corresponding author.

E-mail address: adla@nmr.mpibpc.mpg.de (A. Lange).
}

Zhou et al. applied DREAM [12], that recouples homonuclear dipolar interactions, for polarization transfer. The application of INEPT transfer bears a number of experimental drawbacks. Due to scalar relaxation of the second kind $[15,16]$, the transversal magnetization life time of CA bound to ${ }^{2} \mathrm{H}$ is relatively short in the absence of ${ }^{13} \mathrm{C}^{2} \mathrm{H}$ scalar decoupling. Such scalar decoupling has recently been achieved by Meier and coworkers [17] but in most commercial ssNMR probes the necesseray fourth channel for deuterium is missing. Furthemore, the INEPT sequence comprises 10 selective pulses and due to probe inhomogeneity this can result in poor performance. Even moderate magnetization losses on the order of $10 \%$ for a single pulse result in overall losses of $65 \%$. At spinning rates that can be reached with $3.2 \mathrm{~mm}$ probes $(\sim 25 \mathrm{kHz})$, polarization losses originate also from imperfectly averaged CSAs and from the interference of spinning rate and the applied ${ }^{13} \mathrm{C}$ RF field with an amplitude of $6-8 \mathrm{kHz}$ used for the band-selective pulses. Alternatively, DREAM [18] can be employed in a straightforward manner and it is very efficient, but requires magic-angle spinning rates that are higher then the chemical shift difference between $\mathrm{CO}$ and CA (e.g. $120 \mathrm{ppm}=24 \mathrm{kHz}$ at $800 \mathrm{MHz}$ proton Larmor frequency) [19]. This condition is not always achievable at moderate spinning rates and high magnetic fields.

Here, we present an alternative robust experimental method for dipolar-based CO-CA magnetization transfer that works well at moderate MAS rates (ca. $20 \mathrm{kHz}$ ) that are smaller than the isotropic chemical shift difference between the $\mathrm{CO}$ and CA bands. The method relies on efficient band-selective homonuclear cross-polarization (BSH-CP). Both zero- and double-quantum homonuclear recoupling can be achieved by continuous $\mathrm{RF}$ irradiation, if the Hartmann-Hahn condition is satisfied, i.e. if the sum or difference 
of the effective fields acting on $\mathrm{CO}$ and $\mathrm{CA}$ is equal to one or two times the spinninig rate. Under the experimental conditions of the present study, we obtained efficient CO-CA magnetization transfer when (i) the sum was equal to two times the spinning rate or (ii) when the difference was equal to the spinning rate, respectively. Experimentally, we achieved magnetization transfer rates of up to $50 \%$, allowing us to record 2D CO-CA correlation spectra of highly deuterated PrgI needles with high sensitivity [20]. The described method is conceptually similar to well-known recoupling techniques under continuous RF irradiation such as HORROR [21], DREAM [18], and rotational resonance in the tilted frame [22-24], but uses different recoupling conditions, providing a new tool for efficient band-selective homonuclear magnetization transfer at moderate spinning rates (ca. $20-30 \mathrm{kHz}$ ) and high magnetic fields (ca. 700-900 MHz ${ }^{1} \mathrm{H}$ Larmor frequency).

\section{Materials and methods}

Expression, purification and polymerization of perdeuterated ${ }^{15} \mathrm{~N}$ - and ${ }^{13} \mathrm{C}$-labelled wild-type PrgI protein was performed as described before $[3,20]$. A low degree of protonation of the sample was achieved according to the approach described earlier by Reif and coworkers [6]. Proton back-exchange took place in a buffer containing a mixture of $10 \%{ }^{1} \mathrm{H}_{2} \mathrm{O}$ and $90 \%{ }^{2} \mathrm{H}_{2} \mathrm{O}$. Approximately $12 \mathrm{mg}$ of material were packed into a $3.2 \mathrm{~mm}$ rotor. All experiments were conducted on a $20 \mathrm{~T}$ wide-bore spectrometer (Bruker Biospin, Germany) equipped with an Avance III console and a $\left({ }^{1} \mathrm{H},{ }^{13} \mathrm{C},{ }^{15} \mathrm{~N}\right)$ triple-resonance $3.2 \mathrm{~mm}$ probe. The MAS rate was adjusted to $20 \mathrm{kHz}$ and the effective sample temperature was set to $12 \pm 2{ }^{\circ} \mathrm{C}$ as measured by the temperature-dependent water proton resonance relative to an internal DSS reference.

Hard proton and carbon pulses employed RF field strengths of $70 \mathrm{kHz}$ and $50 \mathrm{kHz}$, respectively. WALTZ-16 [25] decoupling with an RF field strength of $2.5 \mathrm{kHz}$ was applied on the ${ }^{15} \mathrm{~N}$ channel during ${ }^{13} \mathrm{C}$ evolution and detection periods to remove heteronuclear scalar couplings. A contact time of $3.2 \mathrm{~ms}$ and $2.35 \mathrm{~ms}$ was used for the $\mathrm{H}-\mathrm{CO}$ and $\mathrm{H}-\mathrm{CA}$ heteronuclear $\mathrm{CP}$ steps, respectively. A constant proton field strength of $\sim 49 \mathrm{kHz}$ was used, whereas the ${ }^{13} \mathrm{C}$ $\mathrm{RF}$ field strength was ramped down to $80 \%$ from an initial value of $\sim 32 \mathrm{kHz}$.

\section{Theoretical background}

In this section we briefly outline the theory of the spin dynamics of two dipolar-coupled homonuclear spins I and S in a powdered solid under MAS and continuous RF irradiation. A detailed description has been presented elsewhere [22-24]. For the sake of simplicity we neglect the chemical shift anisotropy (CSA). The total spin Hamiltonian ( $\mathrm{SH}$ ) in the rotating frame is given by the sum of the time-dependent dipolar part $H_{D}(t)$ as well as the Zeeman and RF field part $H_{Z}$ :

$H(t)=H_{Z}+H_{D}(t)$

with the Zeeman and RF field interactions:

$H_{Z}=-\Delta \omega_{I} \cdot I_{Z}-\Delta \omega_{S} \cdot S_{Z}-\omega_{1}\left(S_{X}+I_{X}\right)$

where $\Delta \omega_{X}$ is the isotropic chemical shift offset from the carrier frequency for the spin $X(I$ or $S)$. The cartesian components $x, y, z$ of spin operators $S$ and $I$ are represented by $S_{X}, S_{Y}, S_{Z}$ and $I_{X}, I_{Y}, I_{Z}$, respectively. The amplitude of the applied continuous $R F$ irradiation is $\omega_{1}$.

The dipolar interaction in the rotating frame is represented by:

$H_{D}(t)=D(t) \cdot\left[S_{Z} I_{Z}-\frac{1}{4}\left(I_{+} S_{-}+S_{+} I_{-}\right)\right]$ where the spatial part of the dipolar coupling $D(t)$ is periodically modulated by MAS with the spinning rate $\omega_{r}$ and can be expressed as:

$$
\begin{aligned}
& D(t)=\sum_{n= \pm 1, \pm 2} \omega_{D, n} \cdot \exp \left(i \cdot n \omega_{r} t\right) \\
& \omega_{D, \pm 1}=-\frac{\sqrt{2}}{2} \cdot d \cdot \sin \left(2 \theta_{D}\right) \cdot \exp \left( \pm i \cdot \varphi_{D}\right) \\
& \omega_{D, \pm 2}=\frac{1}{2} \cdot d \cdot \sin ^{2}\left(\theta_{D}\right) \cdot \exp \left( \pm 2 i \cdot \varphi_{D}\right)
\end{aligned}
$$

where the polar angles $\theta_{D}$ and $\varphi_{D}$ define the direction of the internuclear $\mathrm{S}-\mathrm{I}$ vector in a rotor-fixed frame and the dipolar coupling constant $d=-\left(\mu_{0} / 4 \pi\right)\left(\gamma^{2} h / r^{3}\right)$ depends on the internuclear distance $r$ and the gyromagnetic ratio $\gamma \cdot \mu_{0}$ and $h$ are the permeability of free space and Planck's constant, respectively.

The effective fields $\omega_{X, \text { eff }}$ for spins I and S are determined by the applied RF field $\omega_{1}$ and the chemical shift offsets $\Delta \omega_{X}$ :

$\omega_{X, e f f}=\left(\omega_{1}^{2}+\Delta \omega_{X}^{2}\right)^{1 / 2}$

and are tilted with respect to the static external magnetic field by the angles:

$\beta_{X}=\arctan \left(\omega_{1} / \Delta \omega_{X}\right)$

Transformation into a tilted rotating frame determined by the effective RF fields can be achieved using the unitary operator:

$U_{1, e f f}=\exp \left(i \beta_{I} \cdot I_{Y}\right) \cdot \exp \left(i \beta_{S} \cdot S_{Y}\right)$

The resulting Zeeman and dipolar terms are then given by:

$$
\begin{aligned}
H_{Z}^{\prime}= & -\omega_{I, e f f} \cdot I_{Z}^{\prime}-\omega_{S, e f f} \cdot S_{Z}^{\prime} \\
H_{D}^{\prime}(t) & =D(t) \cdot\left[A \cdot S_{Z}^{\prime} I_{Z}^{\prime}-\frac{1}{4} B \cdot\left(S_{+}^{\prime} \cdot I_{-}^{\prime}+S_{-}^{\prime} \cdot I_{+}^{\prime}\right)\right. \\
& \left.+\frac{1}{4} Q \cdot\left(S_{+}^{\prime} \cdot I_{+}^{\prime}+S_{-}^{\prime} \cdot I_{-}^{\prime}\right)+R \cdot S_{Z}^{\prime} \cdot I_{ \pm}^{\prime}+P \cdot S_{ \pm}^{\prime} \cdot I_{Z}^{\prime}\right]
\end{aligned}
$$

The geometrical scaling factors $A, B, Q R$ and $P$ have been described elsewhere [22-24]. The relevant ones for zero- and double-quantum recoupling (ZQ and $\mathrm{DQ}$, respectively) are $\mathrm{B}$ and $\mathrm{Q}$ :

$$
\begin{aligned}
& B=\frac{1}{2}\left(1+\cos \beta_{S} \cdot \cos \beta_{I}-2 \sin \beta_{S} \cdot \sin \beta_{I}\right) \\
& Q=\frac{1}{2}\left(1-\cos \beta_{S} \cdot \cos \beta_{I}+2 \sin \beta_{S} \cdot \sin \beta_{I}\right)
\end{aligned}
$$

Transition into the interaction frame by the unitary operator

$U_{2, e f f}(t)=\exp \left(-i \omega_{I, e f f} \cdot I_{Z}^{\prime} t\right) \cdot \exp \left(-i \omega_{S, e f f} \cdot S_{Z}^{\prime} t\right)$

reduces the $\mathrm{SH}$ to the dipolar interaction only. Using the transformation property of raising and lowering operators, $\exp \left(-i \omega \cdot S_{z} t\right) \cdot S_{ \pm} \cdot \exp \left(i \omega \cdot S_{z} t\right)=S_{ \pm} \cdot \exp (\mp i \omega \cdot t)$, one can readily obtain the $\mathrm{SH}$ in the interaction frame:

$$
\begin{aligned}
H_{D}^{\prime \prime}(t)=U_{2, e f f}(t) H_{D}^{\prime}(t) U_{2, e f f}^{-1}= & \left\{\sum_{n= \pm 1, \pm 2} \omega_{D, n} \cdot \exp \left(i n \omega_{r} t\right)\right\} \\
& \times\left\{A \cdot S_{Z}^{\prime \prime} I_{Z}^{\prime \prime}\right. \\
& -\frac{1}{4} B \cdot\left(S_{+}^{\prime \prime} \cdot I_{-}^{\prime \prime} \cdot \exp \left(-i \Delta_{\text {eff }} \cdot t\right)\right. \\
& \left.+S_{-}^{\prime \prime} \cdot I_{+}^{\prime \prime} \cdot \exp \left(i \Delta_{\text {eff }} \cdot t\right)\right) \\
& +\frac{1}{4} Q \cdot\left(S_{+}^{\prime \prime} \cdot I_{+}^{\prime \prime} \cdot \exp \left(-i \Sigma_{\text {eff }} \cdot t\right)\right. \\
& \left.+S_{-}^{\prime \prime} \cdot I_{-}^{\prime \prime} \cdot \exp \left(i \Sigma_{\text {eff }} \cdot t\right)\right) \\
& +R \cdot S_{Z}^{\prime \prime} \cdot I_{ \pm}^{\prime \prime} \cdot \exp \left(\mp i \omega_{I, e f f} \cdot t\right) \\
& \left.+P \cdot S_{ \pm}^{\prime \prime} \cdot I_{Z}^{\prime \prime} \cdot \exp \left(\mp i \omega_{S, e f f} \cdot t\right)\right\}
\end{aligned}
$$


(A)

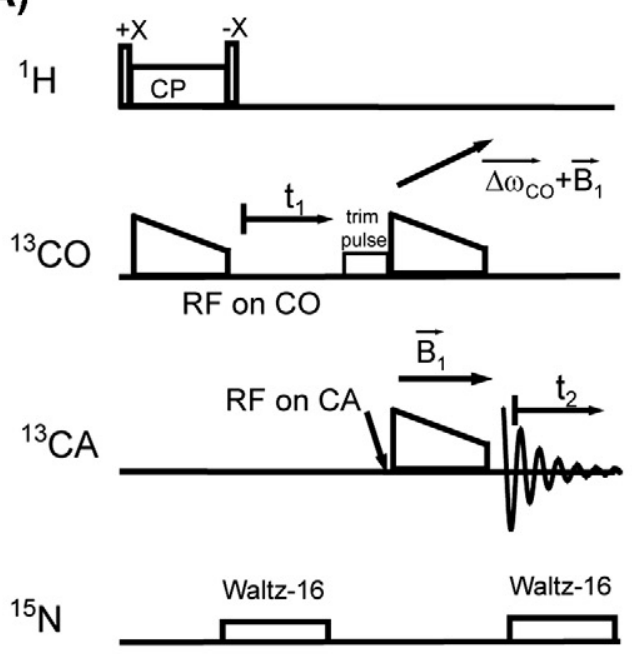

(B)

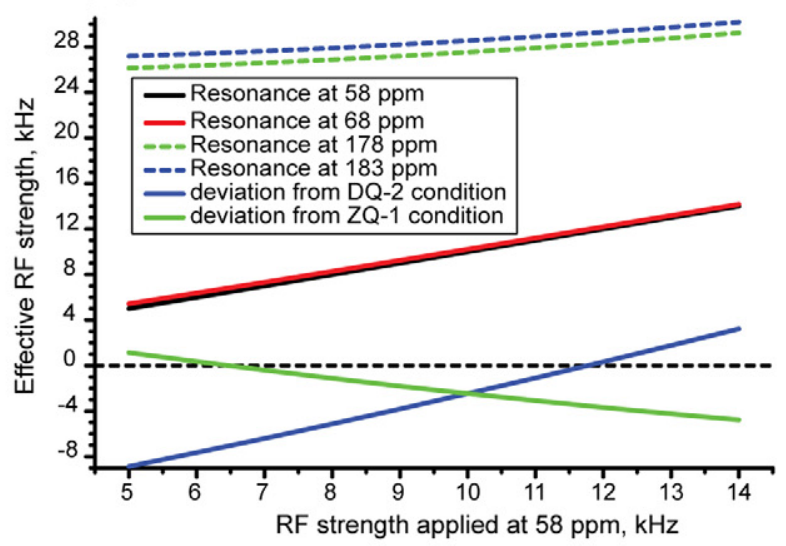

(C)

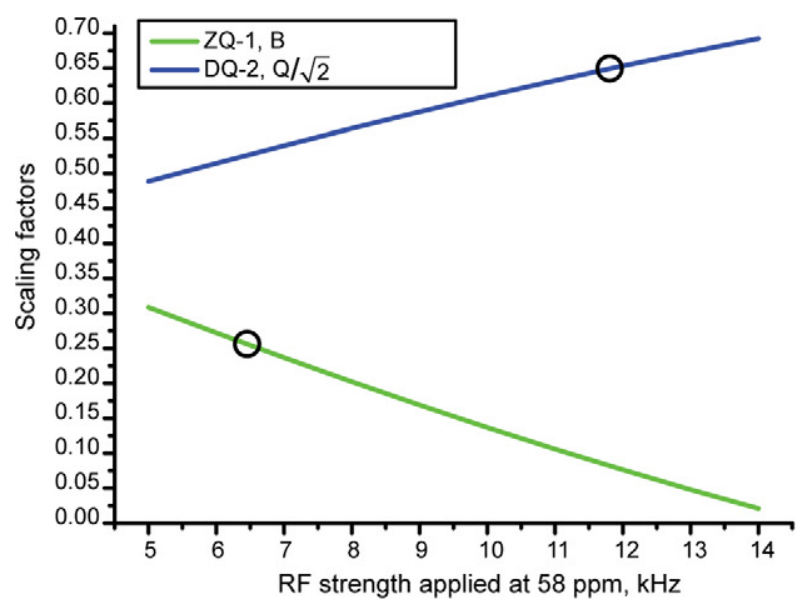

(D)

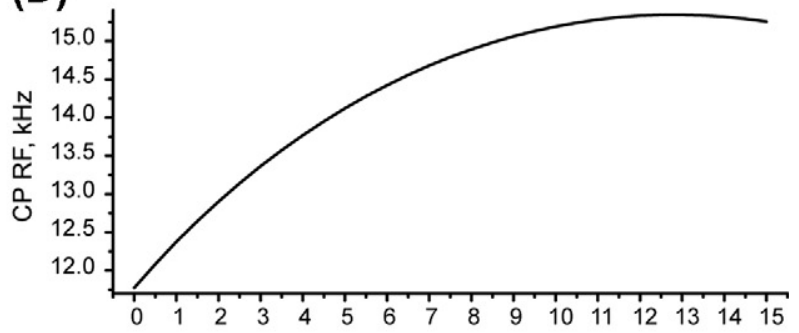

(E)

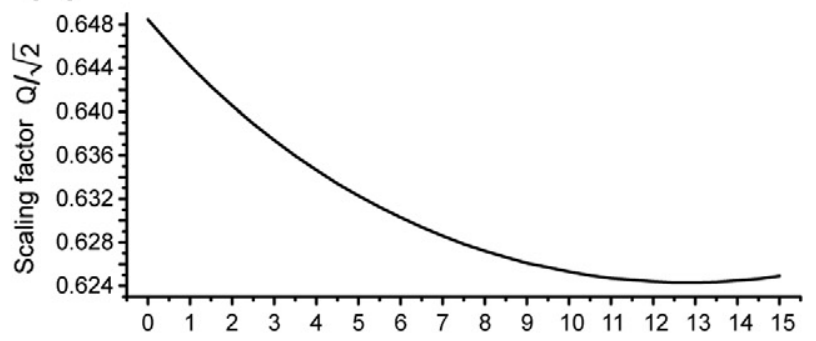

(F)

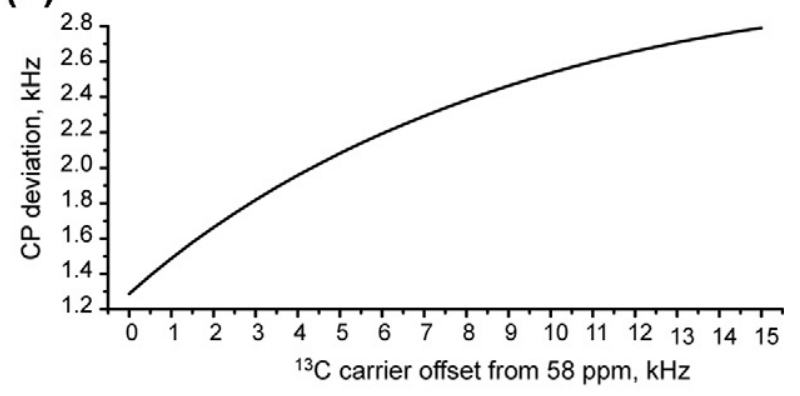

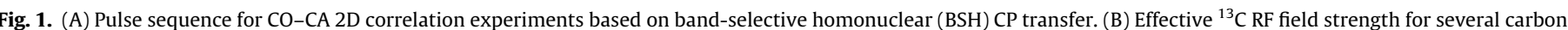

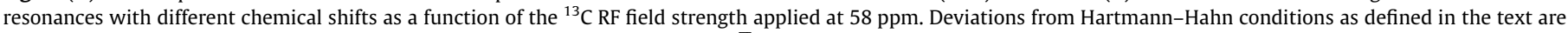

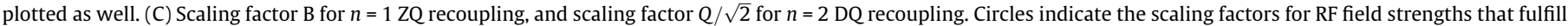

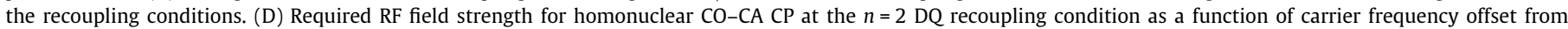

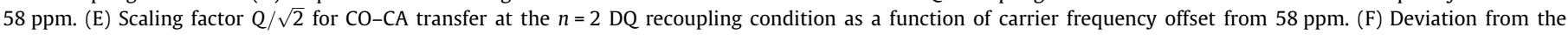
recoupling condition for $n=2$, DQ BSH-CP as defined in the text as a function of carrier frequency offset from 58 ppm.

where the sum and difference of effective fields are given by $\Sigma_{\text {eff }}=$ $\omega_{S, e f f}+\omega_{I, e f f}$ and $\Delta_{\text {eff }}=\omega_{S, e f f}-\omega_{I, e f f}$.
Recoupling takes place at various conditions when the modulations of the spatial and spin parts of the SH mutually cancel each 
other. Here, we are interested in recoupling of the flip-flop and flop-flop terms that correspond to ZQ and DQ transitions, respectively, and which occur at:

$\Delta_{\text {eff }}=\left|\omega_{S, e f f}-\omega_{I, e f f}\right|=n \cdot \omega_{r}$

and

$\Sigma_{\text {eff }}=\omega_{S, e f f}+\omega_{I, e f f}=n \cdot \omega_{r}$

where $n=1,2$.

Recoupling techniques that fulfill Eqs. (14) and (15) are (I) rotational resonance in the tilted frame [22-24] which is a frequencyselective recoupling method and (II) HORROR [21] and DREAM [18] which require that the recoupling $R F$ field strength is set to approximately half of the spinning rate, which directly defines the recoupling band width. These techniques are applicable when the isotropic chemical shift differences between the recoupled spins are considerably smaller than the spinning rate.

Here, we show that homonuclear Hartman-Hahn CP transfer fulfilling also Eqs. (14) and (15) - at moderate MAS rates and at high magnetic fields can be efficiently employed for band-selective CO-CA magnetization transfer in deuterated proteins even though the isotropic chemical shift difference of CO and CA considerably exceeds the spinning rate. As will be shown below, the resulting relatively weak scaling factors for $\mathrm{ZQ}$ and $\mathrm{DQ}$ recoupling entail the use of long CP contact times. Nevertheless, in highly deuterated proteins, experimental drawbacks that would usually be associated with such long contact times such as significant loss of magnetization, sample heating and eventually probe damage, can be avoided because no high power proton decoupling is required.

\section{Experimental results and discussion}

Fig. $1 \mathrm{~A}$ presents the pulse program for 2D CO-CA correlation spectroscopy based on band-selective homonuclear $\mathrm{CP}$ (BSH-CP) transfer. Directly excited proton magnetization is selectively transferred to $\mathrm{CO}$ by $\mathrm{CP}$ followed by a proton flip back (FB) pulse in order to preserve and reuse remaining proton magnetization [26]. Employing a proton FB allows reducing the experimental time by a factor of 2.2. After subsequent chemical shift evolution, the CO magnetization is flipped by a hard trim pulse along the $Z^{\prime}$ axis of the tilted rotating frame determined by the ${ }^{13} \mathrm{C}$ RF field applied on CA for BSH-CP. The flip angle is $\left(90-\beta_{\mathrm{CO}}\right)$ and determined by Eq. (7). Polarization from $\mathrm{CO}$ is transferred to $\mathrm{CA}$ under continuous RF irradiation, chosen to satisfy the $n=2$, DQ recoupling condition for $\mathrm{CO} / \mathrm{CA}$ spin pairs. During ${ }^{13} \mathrm{C}$ evolution and detection periods, WALTZ-16 [25] is applied on the ${ }^{15} \mathrm{~N}$ channel to remove ${ }^{15} \mathrm{~N}-{ }^{13} \mathrm{C}$ scalar couplings. No proton decoupling is used during any stage of the experiment.

The calculation results presented in Fig. 1B-F assume an external magnetic field corresponding to $850 \mathrm{MHz}{ }^{1} \mathrm{H}$ Larmor frequency and an MAS rate of $20 \mathrm{kHz}$. Fig. 1B shows the effective ${ }^{13} \mathrm{C}$ RF field strength for a number of carbon resonances with different chemical shifts as a function of the ${ }^{13} \mathrm{C}$ RF field strength applied at $58 \mathrm{ppm}$ as determined by Eq. (6). The red and black lines represent the effective ${ }^{13} \mathrm{C}$ fields at $58 \mathrm{ppm}$ and $68 \mathrm{ppm}$, respectively. Within the band of the CA resonances the effective fields are not very dependent on the resonance position which can be seen from the fact that the lines lie very close together. The chemical shift variations in the CO band affect the effective RF field more strongly, as demonstrated by the blue and green dashed lines corresponding to resonances at $178 \mathrm{ppm}$ and $183 \mathrm{ppm}$, respectively. The difference is about $1 \mathrm{kHz}$ and can mismatch the BSH-CP conditions for some resonances due to chemical shift dispersion. Solid blue and green lines show the deviation of two resonances at $58 \mathrm{ppm}$ and $178 \mathrm{ppm}$ from ideal DQ, $n=2$ and $\mathrm{ZQ}, n=1$ conditions at different
${ }^{13} \mathrm{C}$ field strengths applied at $58 \mathrm{ppm}$. The calculations were made according to Eqs. (14) and (15). The deviations from DQ, $n=2$ $\left(\Delta \mathrm{CP}_{\mathrm{DQ} 2}\right)$ and $\mathrm{ZQ}, n=1\left(\Delta \mathrm{CP}_{\mathrm{ZQ} 1}\right)$ recoupling conditions were calculated as $\Delta \mathrm{CP}_{\mathrm{DQ} 2}=\omega_{178 \mathrm{ppm}, \text { eff }}+\omega_{58 \mathrm{ppm} \text {,eff }}-2 \omega_{r}$ and $\Delta \mathrm{CP}_{\mathrm{ZQ} 1}=$ $\omega_{178 p p m, e f f}-\omega_{58 p p m, e f f}-\omega_{r}$ respectively. These values show qualitatively how robust the recoupling is with respect to possible RF field variations. The plot shows that $\mathrm{ZQ}$ and $\mathrm{DQ}$ recoupling take place at RF strengths of 6.5 and $11.8 \mathrm{kHz}$, respectively.

The efficiency of the magnetization transfer is determined by the scaling factors of the recoupled interactions, provided by Eq. (11). Green and blue lines in Fig. 1C represent the scaling factor $B$ for ZQ recoupling, $n=1$ and the factor $Q / \sqrt{2}$ for DQ recoupling, $n=2$, respectively, as a function of the applied RF field amplitude. In the latter case we scaled the factor $Q$ by $\sqrt{2}$ to allow for a direct comparison of the considered scaling factors, because the amplitude of the recoupled interaction at conditions with $n=2$ is smaller by $\sqrt{2}$ compared to the amplitude at conditions with $n=1$, which follows from Eq. (5). The low steepness of the lines indicates that the recoupling conditions are robust with respect to RF field amplitude variations.

Fig. 1D shows the required RF field strength for homonuclear CP between two resonances at $58 \mathrm{ppm}$ and $178 \mathrm{ppm}$ as a function of the RF carrier offset from $58 \mathrm{ppm}$ to higher ppm values. Fig. 1E represents the scaling factor $Q / \sqrt{2}$ for the $n=2$, DQ recoupling condition between two resonances at $58 \mathrm{ppm}$ and $178 \mathrm{ppm}$ as a function of RF carrier offset from $58 \mathrm{ppm}$. Fig. $1 \mathrm{~F}$ shows the difference between double MAS rate $\left(2 \omega_{r}=40 \mathrm{kHz}\right)$ and sum of effective fields at $183 \mathrm{ppm}$ and $68 \mathrm{ppm}$ when the applied RF field matches the $\mathrm{DQ} n=2 \mathrm{CP}$ condition for two resonances at $58 \mathrm{ppm}$ and $178 \mathrm{ppm}$. The plot reveals the deviation from the ideal recoupling condition due to possible isotropic chemical shift variations of CA and CO. Fig. 1D and E indicate that both scaling factor and RF field strength for the DQ, $n=2$ recoupling condition are well acceptable at any RF carrier position, while the deviation from the recoupling condition due to chemical shift dispersion is minimal when the RF carrier is set to the center of the CA band, which is imposed by the larger chemical shift dispersion of this carbon type compared to CO.
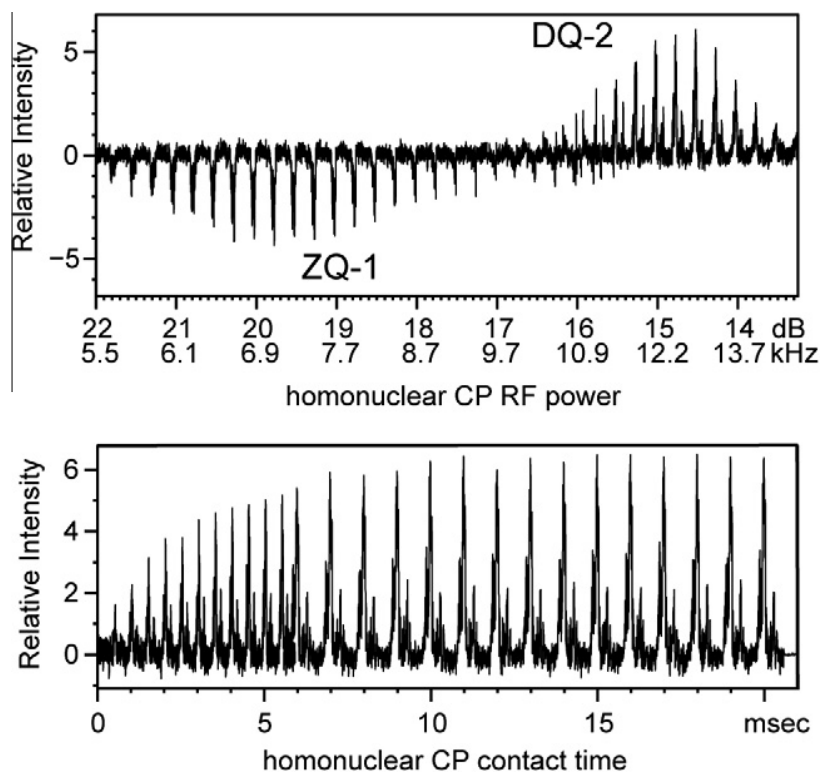

Fig. 2. Top: CA signal after CO-CA BSH-CP as a function of maximal RF field strength applied at $58 \mathrm{ppm}$. Bottom: CA magnetization build-up as a function of contact time during $\mathrm{BSH}-\mathrm{CP}$ at the $n=2 \mathrm{DQ}$ recoupling condition. 
Fig. 2 demonstrates CO-CA homonuclear magnetization transfer as a function of the applied RF field strength (top) and for the $n=2$ DQ recoupling condition as a function of the CP contact time (bottom). One-dimensional experiments were performed according to the pulse scheme shown in Fig. $1 \mathrm{~A}$, with the ${ }^{13} \mathrm{C}$ field ramped down to $80 \%$ amplitude during BSH-CP. The top panel shows magnetization transfer at both recoupling conditions, namely ZQ, $n=1$ and $\mathrm{DQ}, n=2$ at maximum RF field strengths of ca. $7 \mathrm{kHz}$ and $12.8 \mathrm{kHz}$, respectively, in agreement with the theoretical expectations (Fig. 1 and Eqs. (14) and (15)). The trim pulse was set to $70^{\circ}$ during the RF field strength optimization (as shown in the top panel), as a compromise between optimal values of $76^{\circ}$ and $65^{\circ}$ for ZQ and DQ transfer, respectively. A contact time of $14 \mathrm{~ms}$ was used. The optimization reveals that the DQ transfer provides higher transfer efficiency compared to ZQ transfer, which is in line with theoretical expectations concerning the scaling factor and the recoupling bandwidth. The magnetization builds up relatively slowly and reaches a maximum after $11 \mathrm{~ms}$ for DQ $n=2$ recoupling, as plotted in the bottom panel. Notably, such long contact

(A)

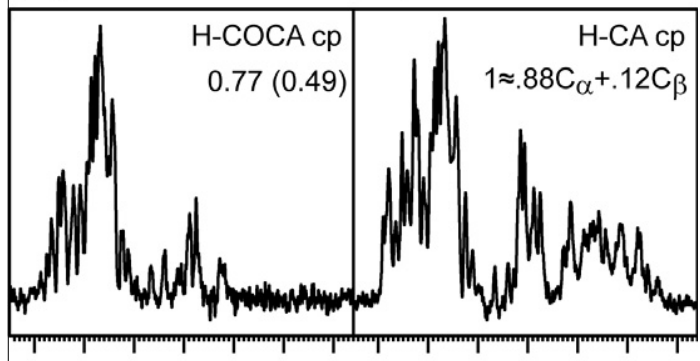

(C)

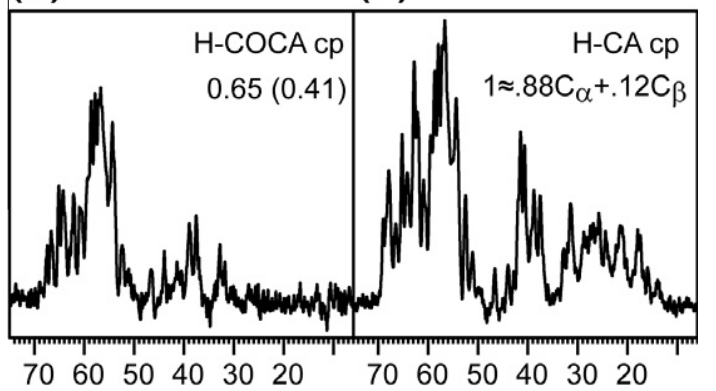

(E)

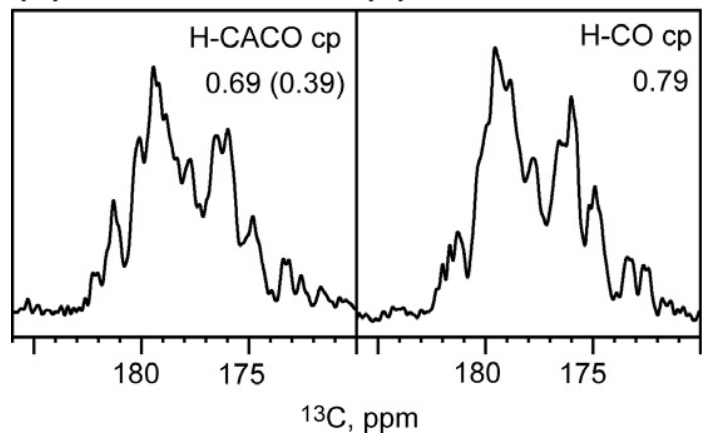

Fig. 3. ( $A, C$ and $E$ ) $C A$ and $C O$ signal after $B S H-C P$ in comparison with (B, D and F) reference heteronuclear $C P$ spectra. The number of scans for homonuclear $C P$ experiments ( $A, C$ and $E$ ) was two times more than for the reference experiments ( $B$, $\mathrm{D}$ and $\mathrm{F})$. The numbers in the panel refer to the integrated signal in the regions 43.3-70.3 ppm (CA) or 168.5-186 ppm (CO); the different number of scans was not accounted for. The estimated efficiency of $\mathrm{BSH}-\mathrm{CP}$ in each experiment is given in parentheses. times are not a severe limitation for the application to highly deuterated proteins, due to the resulting extremely long coherence lifetime in the absence of high-power proton decoupling. Furthermore, the moderately high $\mathrm{RF}$ strength for $\mathrm{DQ} n=2$ recoupling makes the transfer quite robust in terms of hardware instability and RF inhomogeneity. The build-up dynamics are consistent with results from numerical simulations as presented in the subsequent section.

Fig. 3 compares reference $1 \mathrm{D}{ }^{13} \mathrm{C}$ spectra obtained by bandselective $\mathrm{H}-\mathrm{CO}$ or $\mathrm{H}-\mathrm{CA} \mathrm{CP}$ (panels $\mathrm{B}, \mathrm{D}, \mathrm{F}$ ) with spectra obtained by $n=2$, DQ homonuclear $\mathrm{CP}$ following band-selective $\mathrm{H}-\mathrm{CO}$ or $\mathrm{H}-\mathrm{CA}$ CP (panels A, C, E). The 1D version of the pulse scheme in Fig. 1A was employed for the spectra in panels $A$ and $C$. The $C O$ spectrum in panel $\mathrm{E}$ was recorded by means of a straightforward adaptation of the above mentioned pulse sequence, which is depicted in Fig. 1 of the Supporting information (SI). During homonuclear $\mathrm{CP}$ the carbon RF field was ramped down to $70 \%$ in $\mathrm{C}$ and $\mathrm{E}$ and to $80 \%$ in A. Panels B, D and F give 1D CA and CO reference signals obtained by standard band-selective heteronuclear ${ }^{1} \mathrm{H}-{ }^{13} \mathrm{CA} /{ }^{13} \mathrm{CO} \mathrm{CP}$. The same experimental spectrum is plotted in $B$ and $D$. The number of scans for BSH-CP experiments in $A, C$ and $\mathrm{E}$ was two times more then for the reference spectra in $\mathrm{B}, \mathrm{D}$ and $\mathrm{F}$. For the maximal ${ }^{13} \mathrm{C}$ RF field strength that was ramped down to $80 \%$ and $70 \%$ during $\mathrm{BSH}-\mathrm{CP}$ optimal values of $12.8 \mathrm{kHz}$ and $14.4 \mathrm{kHz}$ were found and the optimum contact times were $11.4 \mathrm{~ms}$ and $13.5 \mathrm{~ms}$, respectively. Fig. 3 reveals that the carbon signal is smaller by factor of $2-2.5$ in the homonuclear CP spectra compared to the reference spectra. The data also show the advantage of using a less steep ramp on the ${ }^{13} \mathrm{C}$ RF field. Integral values for the regions $43.3-70.3 \mathrm{ppm}$ or $168.5-186 \mathrm{ppm}$ are provided in the panels, without accounting for the number of scans. The values in parentheses refer to the estimated efficiency of homonuclear CP. This is given by the ratio of the integral value of the current spectrum and the integral value of the reference spectrum of the carbon

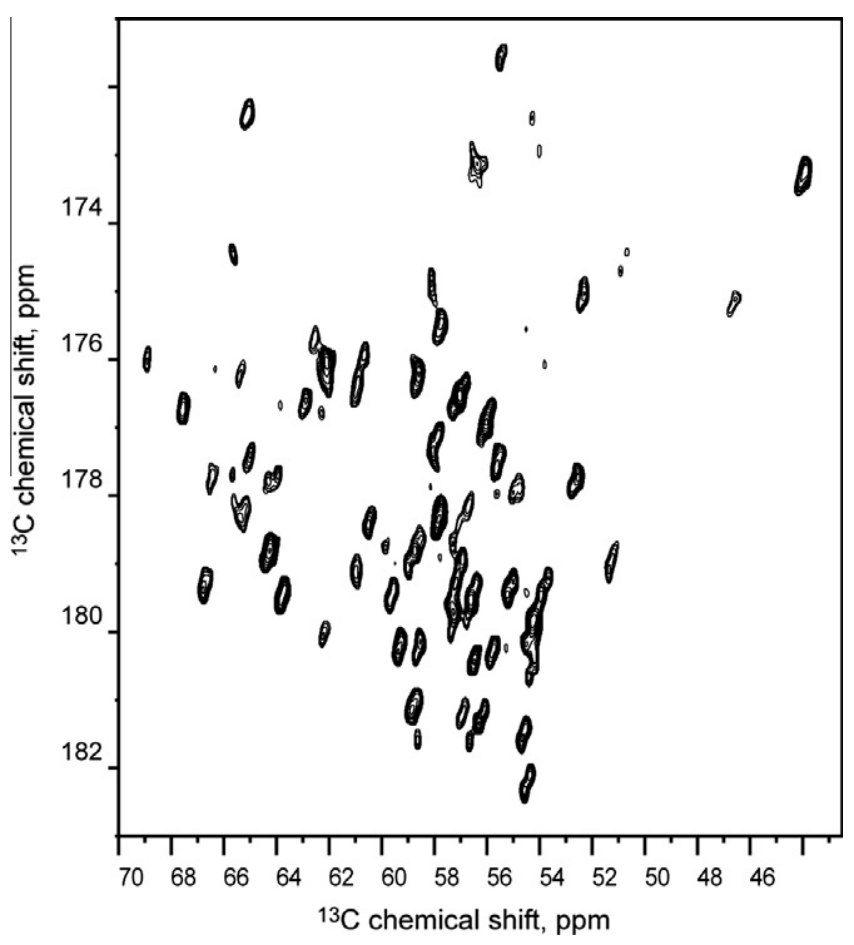

Fig. 4. A $2 D$ CO-CA correlation spectrum of $U-\left[{ }^{2} \mathrm{H},{ }^{13} \mathrm{C},{ }^{15} \mathrm{~N}\right]$-labeled type three secretion system (T3SS) needles (at 10\% back-exchange of amide protons). The CO$\mathrm{CA}$ transfer relied on $\mathrm{BSH}-\mathrm{CP}$ at the $n=2 \mathrm{DQ}$ recoupling condition. The spectrum was recorded on an $850 \mathrm{MHz}$ spectrometer at $20 \mathrm{kHz}$ MAS. 
region, that was polarized prior homonuclear CP transfer. To calculate the efficiency we normalized the integral values by the number of scans used. In detail, the values from the following panels were compared: $A$ and $\mathrm{F}, \mathrm{C}$ and $\mathrm{F}, \mathrm{E}$ and $\mathrm{B}$. The $\mathrm{CA}$ region after heteronuclear $\mathrm{CP}$ contains $11 \mathrm{CB}$ resonances of Ser and Thr, so we estimated that $88 \%$ of the intensity in the spectrum corresponds to $C A$ resonances by assuming equal polarization of $C A$ and $C B$ and no polarization of the flexible residues M1 and A2 [20]. We neglected a possible contribution of the side chain signals of Asp, Asn, Glu and $\mathrm{Gln}$ to the $\mathrm{CO}$ region after $\mathrm{H}-\mathrm{CO} \mathrm{CP}$. In any case, including this contribution into the analysis would only increase the estimated efficiency of CO-CA transfer. The presented results show a high efficiency of up to $50 \%$ for BSH-CP transfer which suggests its use as a building block in $\mathrm{N}_{\mathrm{i}}-\mathrm{CO}_{i-1}-\mathrm{CA}_{i-1}$ experiments that are of paramount importance in protein resonance assignment.

Fig. 4 shows a CO-CA correlation spectrum acquired within $35 \mathrm{~h}$ according to the pulse scheme presented in Fig. 1A. For the BSH-CP transfer, ${ }^{13} \mathrm{C}$ RF power was applied during $11.4 \mathrm{~ms}$

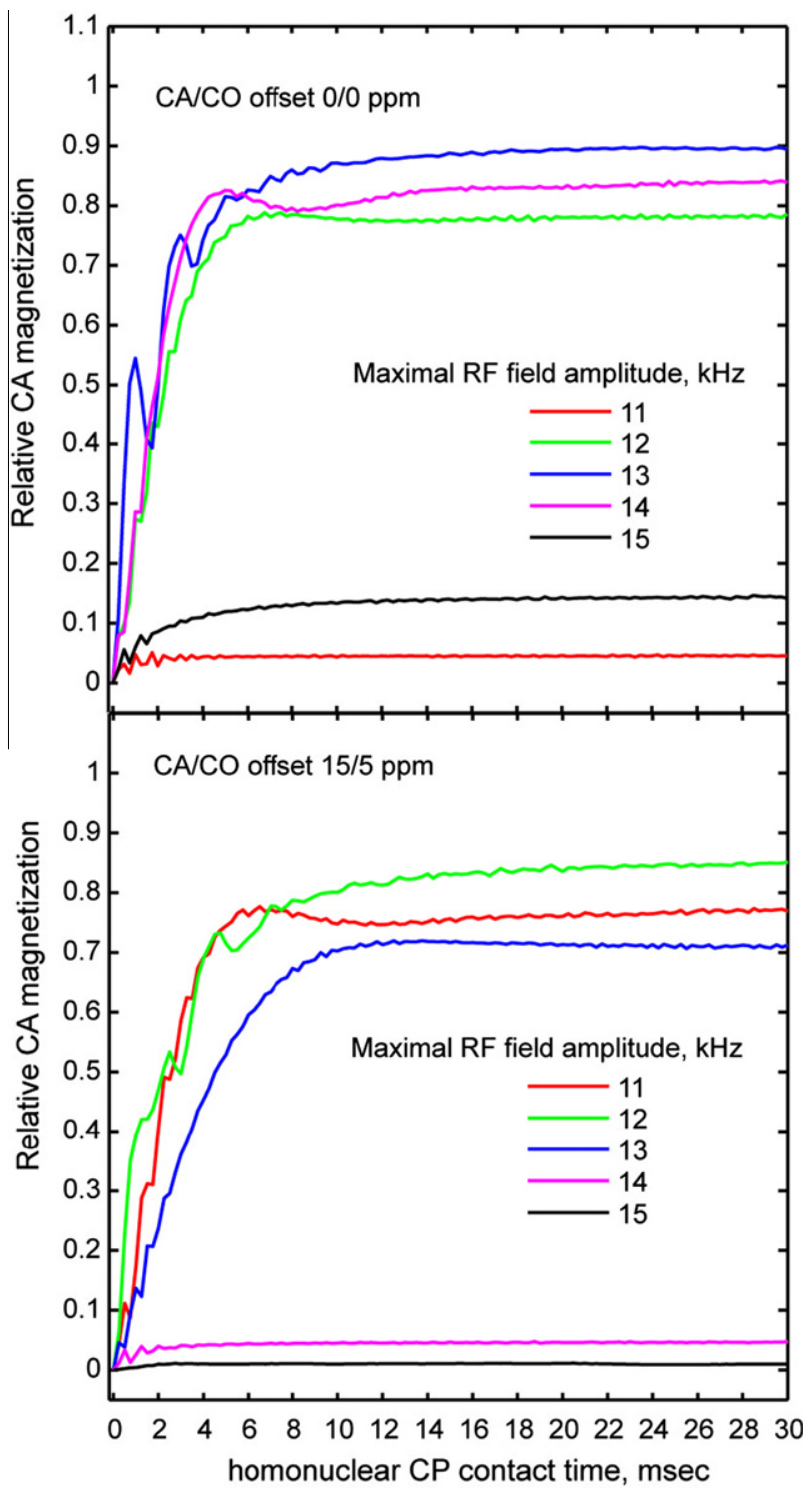

Fig. 5. CA magnetization dynamics during BSH-CP at different RF field strengths, as indicated in the figure. The top panel represents simulation results for CA and CO spins shifted by $1 \mathrm{ppm}$ and $120 \mathrm{ppm}$ from the RF carrier frequency at $0 \mathrm{ppm}$, the bottom panel shows results for CA and CO resonances at $16 \mathrm{ppm}$ and $125 \mathrm{ppm}$, respectively. at $\sim 58 \mathrm{ppm}$ and ramped down to $80 \%$ from an initial RF strength of $\sim 12.8 \mathrm{kHz}$. Maximum acquisition times were $19.8 \mathrm{~ms}$ and $15.75 \mathrm{~ms}$ for the direct and the indirect dimensions, respectively. Prior to Fourier-transformation the data were apodized in both dimensions with a $45^{\circ}$-shifted squared sine bell window function. The spectrum demonstrates excellent signal to noise and resolution. Also the fact that it exhibits cross peaks from the edges of the $\mathrm{CA}$ and $\mathrm{CO}$ bands, demonstrates the broad recoupling bandwidth.

\section{Numerical simulations}

In order to investigate the dynamics of magnetization transfer during $\mathrm{BSH}-\mathrm{CP}$ at variations of ${ }^{13} \mathrm{C}$ isotropic chemical shift and $\mathrm{RF}$ field strength we performed SIMPSON [27] simulations for a spin system comprising a directly bound $\mathrm{CO}$ and CA pair with an internuclear distance of $1.53 \AA$. This corresponds to a dipolar coupling of $2121 \mathrm{~Hz}$. For comparison with the experimental data, $20 \mathrm{kHz}$ MAS and an external magnetic field corresponding to $850 \mathrm{MHz}$ proton Larmor frequency were used. Other parameters of the system and calculations are given in the SI (spin system section of SIMPSON input file).

Fig. 5 shows the CA magnetization build-up as a function of contact time. The initial magnetization of $\mathrm{CO}$ is calibrated to 1 . The simulation describes the pulse sequence of Fig. $1 \mathrm{~A}$ with the ${ }^{13} \mathrm{C}$ $\mathrm{RF}$ field ramped down to $80 \%$. The curves are color coded according to maximal RF amplitudes, as given in the figures. The top panel shows simulation results for CA and CO spins shifted by $1 \mathrm{ppm}$ and $120 \mathrm{ppm}$ from the carrier frequency at $0 \mathrm{ppm}$, while the bottom panel shows results for resonances at $16 \mathrm{ppm}$ and $125 \mathrm{ppm}$. More simulation results can be found in the Supporting information, e.g. for other sets of chemical shifts, as well as for a ${ }^{13} \mathrm{C}$ RF field ramped down to $70 \%$ during $\mathrm{BSH}-\mathrm{CP}$. In summary, the simulations show that $\mathrm{BSH}-\mathrm{CP}$ is robust with respect to probe inhomogeneity and chemical shift dispersion. The comparison to the experimentally obtained data (see Fig. 2) reveals similar magnetization transfer dynamics, in particular regarding the build-up time.

\section{Conclusions}

We could show that band-selective homonuclear CP (BSH-CP) can be applied for efficient polarization transfer between $\mathrm{CO}$ and CA regions in highly deuterated proteins at moderate MAS rates and high magnetic fields. The most efficient recoupling is achieved when the sum of effective RF fields on CA and CO resonances is equal to two times the spinning rate. This method can be directly implemented in proton-detected variants of inter-residual correlation experiments without the requirement of ultra-fast MAS.

\section{Acknowledgments}

This work was supported by the Max Planck Society, the DFG (Emmy Noether Fellowship to A.L.), and the European Union Seventh Framework Program under Grant Agreement 261863 (BioNMR).

\section{Appendix A. Supplementary material}

Supplementary data associated with this article can be found, in the online version, at http://dx.doi.org/10.1016/j.jmr.2013.02.021.

\section{References}

[1] A. McDermott, Structure and dynamics of membrane proteins by magic angle spinning solid-state NMR, Annu. Rev. Biophys. 38 (2009) 385-403. 
[2] C. Wasmer, A. Lange, H. Van Melckebeke, A.B. Siemer, R. Riek, B.H. Meier Amyloid fibrils of the HET-s(218-289) prion form a beta solenoid with a triangular hydrophobic core, Science 319 (2008) 1523-1526.

[3] A. Loquet, N.G. Sgourakis, R. Gupta, K. Giller, D. Riedel, C. Goosmann, C. Griesinger, M. Kolbe, D. Baker, S. Becker, A. Lange, Atomic model of the type III secretion system needle, Nature 486 (2012) 276-279.

[4] V. Chevelkov, U. Fink, B. Reif, Quantitative analysis of backbone motion in proteins using MAS solid-state NMR spectroscopy, J. Biomol. NMR 45 (2009) 197-206.

[5] P. Schanda, B.H. Meier, M. Ernst, Quantitative analysis of protein backbone dynamics in microcrystalline ubiquitin by solid-state NMR spectroscopy, J. Am. Chem. Soc. 132 (2010) 15957-15967.

[6] V. Chevelkov, K. Rehbein, A. Diehl, B. Reif, Ultrahigh resolution in proton solidstate NMR spectroscopy at high levels of deuteration, Angew. Chem., Int. Ed. 45 (2006) 3878-3881.

[7] V. Chevelkov, B.J.V. Rossum, F. Castellani, K. Rehbein, A. Diehl, M. Hohwy, S. Steuernagel, F. Engelke, H. Oschkinat, B. Reif, H-1 detection in MAS solid-state NMR spectroscopy of biomacromolecules employing pulsed field gradients for residual solvent suppression, J. Am. Chem. Soc. 125 (2003) 7788-7789.

[8] E.K. Paulson, C.R. Morcombe, V. Gaponenko, B. Dancheck, R.A. Byrd, K.W. Zilm Sensitive high resolution inverse detection NMR spectroscopy of proteins in the solid state, J. Am. Chem. Soc. 125 (2003) 15831-15836.

[9] D.H. Zhou, J.J. Shea, A.J. Nieuwkoop, W.T. Franks, B.J. Wylie, C. Mullen, D. Sandoz, C.M. Rienstra, Solid-rate protein-structure determination with protondetected triple-resonance 3D magic-angle-spinning NMR spectroscopy, Angew. Chem., Int. Ed. 46 (2007) 8380-8383.

[10] M. Huber, S. Hiller, P. Schanda, M. Ernst, A. Bockmann, R. Verel, B.H. Meier, A proton-detected 4D solid-state NMR experiment for protein structure determination, ChemPhysChem 12 (2011) 915-918.

[11] R. Linser, B. Bardiaux, V. Higman, U. Fink, B. Reif, Structure calculation from unambiguous long-range amide and methyl (1)H-(1)H distance restraints for a microcrystalline protein with MAS solid-state NMR spectroscopy, J. Am. Chem. Soc. 133 (2011) 5905-5912.

[12] D.H. Zhou, A.J. Nieuwkoop, D.A. Berthold, G. Comellas, L.J. Sperling, M. Tang, G. Shah, E.J. Brea, L.R. Lemkau, C.M. Riensta, Solid-state NMR analysis of membrane proteins and protein aggregates by proton detected spectroscopy, J. Biomol. NMR 54 (2012) 291-305.

[13] R. Linser, U. Fink, B. Reif, Proton-detected scalar coupling based assignment strategies in MAS solid-state NMR spectroscopy applied to perdeuterated proteins, J. Magn. Reson. 193 (2008) 89-93.
[14] M.J. Knight, A.L. Webber, A.J. Pell, P. Guerry, E. Barbet-Massin, I. Bertini, I.C. Felli, L. Gonnelli, R. Pierattelli, L. Emsley, A. Lesage, T. Herrmann, G. Pintacuda, Fast resonance assignment and fold determination of human superoxide dismutase by high-resolution proton-detected solid-state MAS NMR spectroscopy, Angew. Chem., Int. Ed. 50 (2011) 11697-11701.

[15] A. Abragam, Principles of Nuclear Magnetism, At the Clarendon Press, 1961.

[16] R.E. London, A theoretical evaluation of the significance of scalar relaxation in coupled spin-1/2 systems in macromolecules, J. Magn. Reson. 86 (1990) 410415.

[17] M. Huber, O. With, P. Schanda, R. Verel, M. Ernst, B.H. Meier, A supplementary coil for H-2 decoupling with commercial HCN MAS probes, J. Magn. Reson. 214 (2012) 76-80.

[18] R. Verel, M. Ernst, B.H. Meier, Adiabatic dipolar recoupling in solid-state NMR: the DREAM scheme, J. Magn. Reson. 150 (2001) 81-99.

[19] J.-P. Demers, V. Chevelkov, A. Lange, Progress in correlation spectroscopy at ultra-fast magic-angle spinning: basic building blocks and complex experiments for the study of protein structure and dynamics, Solid State Nucl. Magn. Reson. 40 (2011) 101-113.

[20] A. Loquet, G. Lv, K. Giller, S. Becker, A. Lange, C-13 spin dilution for simplified and complete solid-state NMR resonance assignment of insoluble biological assemblies, J. Am. Chem. Soc. 133 (2011) 4722-4725.

[21] N.C. Nielsen, H. Bildsoe, H.J. Jakobsen, M.H. Levitt, Double-quantum homonuclear rotary resonance: efficient dipolar recovery in magic-angle spinning nuclear magnetic resonance, J. Chem. Phys. 101 (1994) 18051812.

[22] V. Ladizhansky, R.G. Griffin, Band-selective carbonyl to aliphatic side chain C13-C-13 distance measurements in U-C-13, N-15-labeled solid peptides by magic angle spinning NMR, J. Am. Chem. Soc. 126 (2004) 948-958.

[23] K. Takegoshi, K. Nomura, T. Terao, Rotational resonance in the tilted rotatingframe, Chem. Phys. Lett. 232 (1995) 424-428.

[24] K. Takegoshi, K. Nomura, T. Terao, Selective homonuclear polarization transfer in the tilted rotating frame under magic angle spinning in solids, J. Magn. Reson. 127 (1997) 206-216.

[25] A.J. Shaka, J. Keeler, T. Frenkiel, R. Freeman, An improved sequence for broadband decoupling - WALTZ-16, J. Magn. Reson. 52 (1983) 335-338.

[26] J. Tegenfeldt, U. Haeberlen, Cross polarization in solids with flip-back of I-spin magnetization, J. Magn. Reson. 36 (1979) 453-457.

[27] M. Bak, J.T. Rasmussen, N.C. Nielsen, SIMPSON: a general simulation program for solid-state NMR spectroscopy, J. Magn. Reson. 147 (2000) 296-330. 